\title{
Clinical relevance of telomerase activity in primary gastric lymphoma
}

\author{
Yasuhiro Kodera ${ }^{1}$, Tsuneya Nakamura ${ }^{2}$, Takashi Suzuki ${ }^{2}$, Yoshitaka Yamamura ${ }^{1}$, and Shigeo Nakamura ${ }^{3}$ \\ ${ }^{1}$ Department of Gastroenterological Surgery, Aichi Cancer Center, 1-1 Kanokoden, Chikusa-ku, Nagoya, Aichi 464-8681, Japan \\ ${ }^{2}$ Department of Gastroenterology, Aichi Cancer Center, Aichi, Japan \\ ${ }^{3}$ Department of Pathology, Aichi Cancer Center, Aichi, Japan
}

\begin{abstract}
Background. To distinguish between low-grade lymphoma and reactive lymphoid infiltrate can be demanding for pathologists. Demonstration of B-cell monoclonality by polymerase chain reaction (PCR) analysis of immunoglobulin heavy chain is useful as referential data. Telomerase activity, which is frequently detected in malignant tumors while being undetectable in normal tissues, may also have a supportive role in the diagnostic procedure, but has not been investigated in specimens of primary gastric lymphoma.

Methods. Telomerase activity was qualitatively and quantitatively evaluated, using fluorescence-based telomeric amplification assay protocol (TRAP) analysis, in 16 malignant lymphoma and related specimens, including 7 specimens of low-grade mucosa-associated lymphoid tissue (MALT) lymphoma, among which specimens before and after Helicobacter pylori eradication were evaluated from five patients.

Results. Telomerase activity was detected more frequently (6 of 7 specimens) at higher levels in high-grade lymphoma compared with low-grade MALT lymphoma. It was detected in 3 of 7 specimens of low-grade MALT lymphoma, but was undetectable either at the stage of Helicobacter pyloriinduced gastritis or after $\mathrm{H}$. pylori eradication therapy.

Conclusion. Telomerase activity evaluated by fluorescencebased TRAP analysis was detectable late in the process of evolution of MALT lymphoma and was undetectable early in the process of regression. It was frequently and overtly elevated in high-grade lymphoma. Telomerase activity may have a role in confirming the histologic diagnosis of primary gastric lymphoma.
\end{abstract}

Key word MALT lymphoma of the stomach · High-grade lymphoma $\cdot$ Helicobacter pylori $\cdot$ TRAP assay

Offprint requests to: Y. Kodera

Received: March 16, 2000 / Accepted: May 16, 2000

\section{Introduction}

Primary gastric lymphoma is a relatively uncommon disease, accounting for only $2 \%$ of all malignancies of the stomach treated at Aichi Cancer Center, Japan [1]. Surgery had been the treatment of choice, and excellent treatment results with surgery alone [1] or with surgery plus postoperative chemotherapy [2] have been reported. However, the effectiveness of chemotherapy and the morbidity and impaired quality of life associated with surgery have recently prompted investigators in the West to consider primary chemotherapy with radiation as a first-line treatment for high-grade gastric lymphoma $[3,4]$. The emergence of the concept of lymphoma of mucosa-associated lymphoid tissue (MALT) [5,6] and the knowledge concerning its association with Helicobacter pylori [7] has further complicated the issue of identifying an adequate therapeutic strategy for primary gastric lymphoma. Eradication of $H$. pylori, with a combination of antibiotics plus omeprazole, often leads to regression in both endoscopic and microscopic findings of low-grade MALT lymphoma, and is currently a first-line treatment for this disease entity [8]. These findings suggest that low-grade MALT lymphoma and high-grade lymphoma may represent two distinct disease entities. However, it is also well known that high-grade and low-grade components often co-exist in the same lesion of primary gastric lymphoma [1,9], and there is a hypothesis now that some high-grade lymphoma may evolve from a low-grade predecessor.

$H$. pylori infection often leads to the accumulation of lymphoid tissue in the mucosa, within which B-cell follicles are characteristically present, and this may eventually result in the evolution of MALT lymphoma. A state in which there are various degrees of reactive lymphoid infiltrate may exist before the emergence of the malignant neoplasm, and discrimination between these and true MALT lymphoma through morphol- 
ogical findings in biopsy specimens alone could be quite demanding for pathologists. The demonstration of Bcell monoclonality with polymerase chain reaction (PCR)-mediated analysis of immunoglobulin heavy chain can provide a useful confirmation of the histologic impression of lymphoma [10]. However, the correct interpretation of immunoglobulin heavy chain analysis remains unclear when the PCR turns out to be negative despite histologic findings of MALT lymphoma. Other new methods that provide relevant information to facilitate diagnosis through conventional histologic findings are eagerly awaited.

The enzyme telomerase is a ribonucleoprotein complex that synthesizes a specific repetitive nucleotide sequence onto the ends of telomeres, preventing their shortening [11]. The activation of this enzyme is considered to be responsible for preventing cellular senescence and for the development of cancer [12]. It is active in embryonal and germline cells but remains silent in the majority of differentiated somatic cells. Telomerase activity, therefore, is detected in cancer tissues [13-15], while being undetectable in their noncancerous counterparts, and this activity has been used for discrimination between benign and malignant neoplasms of various origins [16-18]. Some investigators have even confirmed the value of telomerase activity as a prognostic factor $[14,19]$. These findings prompted us to quantitate telomerase activity in surgically resected and biopsy specimens from primary gastric lymphoma. The relevance of telomerase activity in the diagnosis and follow-up, especially of low-grade MALT lymphoma, is discussed.

\section{Subjects and methods}

\section{Clinical samples}

Sixteen consecutive patients with primary gastric lymphoma or related lesions diagnosed at Aichi Cancer Center between May 1997 and April 1999 were enrolled. The research was carried out according to the principles set out in the Declaration of Helsinki 1975. Informed consent was obtained from all the patients involved, and the ethical review board of Aichi Cancer Center Hospital had approved the study. Biopsy samples from the patients with low-grade MALT lymphoma were assessed by an experienced pathologist (S.N.), using established criteria for the diagnosis [5]. To assess alterations in histologic appearances in subsequent biopsies, the confidence of a diagnosis of lymphoma was objectively scored, using a previously published system, in which grades 4 and 5 are considered neoplastic [7]. The diagnosis of surgically resected specimens was made on the basis of histopathologic examination by the same pathologist after the sections had been fixed in $10 \%$ formalin, embedded in paraffin, and routinely stained with Giemsa, hematoxylin-eosin, periodic acid-Schiff, and Gomori silver impregnation. At the same time, the $\mathrm{T}$ - and B-cell nature of the infiltrates was tested with a panel of antibodies applicable to paraffin-embedded sections, including L26/CD20, UCHIL1/CD45RO, CD3, and CD79a (Dako Japan, Kyoto, Japan); CD5, and CD10 (Novocastra Laboratories, Newcastle, United Kingdom); and MT1/CD43 (BioScience Products, Emmenbrucke, Switzerland), using the indirect immunoperoxidase technique.

Seven patients had low-grade MALT lymphoma (grade 5), and H. pylori infection was confirmed in six of these patients by the methods described below, while two patients had H. pylori-induced gastritis (grade 3 ). These two patients presented with endoscopic findings suggestive of MALT lymphoma, but were diagnosed otherwise on the basis of histologic findings of the biopsy specimens. All nine of these patients received antibiotic therapy, as described below, and $H$. pylori was successfully eradicated in all of them. Biopsy specimens before and after $H$. pylori eradication were available for five of the patients with MALT lymphoma and for both of the patients with grade 3 gastritis. Two of the nine patients eventually underwent gastrectomy, because the endoscopic findings of gastric tumor remained unchanged. Surgically resected specimens were available from these two patients for the evaluation of telomerase activity.

Seven patients had high-grade lymphoma of various types. Gastrectomy was performed in five of these patients before they received chemotherapy (four of these patients had diffuse large-cell type and one had follicular lymphoma), while only biopsy specimens were available for the remaining two patients (one with diffuse large-cell type and the other with T-cell lymphoma) who were treated with primary chemotherapy. In each patient who underwent endoscopy, biopsy specimens were taken from lymphoma and morphologically normal mucosa for use in this study, in addition to the specimens required for the routine histopathologic examination. In the patients who underwent surgery, samples were also taken from the tumor and morphologically normal mucosa. All the specimens were snap-frozen in liquid nitrogen and stored at $-80^{\circ} \mathrm{C}$ until use. In addition, samples of cancer and normal mucosa were collected from the surgically resected specimens of three patients with gastric carcinoma, for the comparison of telomerase activities with those of lymphoma tissues.

\section{Eradication of $\mathrm{H}$. pylori}

Culture of the biopsy specimens was performed to confirm the presence of $H$. pylori infection. $H$. pylori 
infection was also determined by testing for the presence of anti- $H$. pylori IgG antibody, using an enzymelinked immunoassay kit (Kyowa Medix, Tokyo, Japan). The patients were then treated for eradication of $H$. pylori by the oral administration of amoxicillin, clarithromycin, and lansoprazole for 2 weeks. Each patient underwent endoscopy 1 and 3 months after completion of the anti-H. pylori therapy, and biopsies were taken on both occasions. Cultures and ${ }^{13} \mathrm{C}$-urea breath test were performed to confirm the eradication of H. pylori.

\section{Telomerase assay}

Telomerase activity was qualitatively and quantitatively evaluated using fluorescence-based telomeric amplification assay protocol (TRAP) analysis [20], using a TRAP-eze Telomerase Detection Kit (Oncor, Gaithersburg, MD, USA). In brief, $10 \mathrm{mg}$ of frozen sample was homogenized in $200 \mu \mathrm{l}$ of icecold 3-[(3-cholamidopropyl)-dimethylammonio]-1propanesulfonate (CHAPS) lysis buffer, and incubated for $30 \mathrm{~min}$ on ice. After incubation, the lysates were centrifuged at $12000 \mathrm{~g}$ for $20 \mathrm{~min}$ at $4{ }^{\circ} \mathrm{C}$. The supernatants were rapidly frozen and stored at $-80^{\circ} \mathrm{C}$. The concentration of protein was determined with the Coomassie Protein Assay Reagent (Pierce Chemical, Rockford, IL, USA), and an aliquot of extract containing $1 \mu \mathrm{g}$ of protein was used for each TRAP assay. Aliquots of extracts were incubated with $0.1 \mathrm{ng}$ Cy-5 labelled telomerase substrate (TS) primer (5'AATCCGTC GAGCAGAGTT-3') in Master Mix (TRAP-eze). After 30-min incubation at $30^{\circ} \mathrm{C}$ and 5-min incubation at $95^{\circ} \mathrm{C}$ to inactivate the telomerase, 30 cycles of PCR was performed at $94^{\circ} \mathrm{C}$ for $30 \mathrm{~s}, 60^{\circ} \mathrm{C}$ for 30 cycles, and $72^{\circ} \mathrm{C}$ for $45 \mathrm{~s}$. The products were diluted with an equal volume of formamide dye solution, heated at $94^{\circ} \mathrm{C}$ for $5 \mathrm{~min}$, and applied ( $\left.5 \mu \mathrm{l} / \mathrm{lane}\right)$ to $10 \%$ denaturing gel, containing $6 \mathrm{M}$ urea, fitted to an automated DNA sequencer (ALF red DNA Sequencer, Pharmacia Biotech, Uppsala, Sweden). During electrophoresis at $45 \mathrm{~W}$, the temperature of the gel was kept at $45^{\circ} \mathrm{C}$.

The data from the ALF red DNA Sequencer were collected and analyzed automatically with Fragment Manager VI.I (Pharmacia Biotech). Each peak, including that of the internal control TSK1 (36bp), was quantified in terms of size, peak height, and peak area. A human hepatocellular carcinoma-derived cell line, HepG2 [21], was used as a positive control. The values for telomerase activity based on the above data were determined by the following formula:

[Total of peak areas in the specimen (peaks at 50bp, $56 \mathrm{bp}, 62 \mathrm{pb}$, etc.] / [peak area of internal control (at $36 \mathrm{bp}$ ) in the specimen] $\div$ [total of peak areas of telomerase activity in HepG2] / [peak are a of internal control in HepG2] $\times 100=$ TPG (total product generated) units

The linearity of this method has been shown to be dependent on the number of HepG2 cells [20]. Telomerase activity was clearly detected with a sample containing ten HepG2 cells.

\section{Results}

\section{Effect of $\mathrm{H}$. pylori eradication}

H. pylori was successfully eradicated in all six patients with $H$. pylori-positive MALT lymphoma and in the two patients with reactive gastritis. Eradication of $H$. pylori resulted in histological and endoscopic regression in four of the six H. pylori-positive MALT lymphoma patients, while no response was observed in the two other patients, who were subsequently treated with surgical resection. One patient with low-grade MALT lymphoma received antibiotic therapy, despite the absence of $H$. pylori by the conventional methods of detection, but the therapy did not result in histological or endoscopic regression.

\section{Detection of telomerase activity in specimens of gastric lesions}

In the telomerase-positive samples, telomerase activity was detected as a shark-toothed pattern starting from the first amplifiable telomerase product at $50 \mathrm{bp}$, with a periodicity of about six nucleotides. Extracts from all telomerase-negative samples gave a positive signal for the internal control TSK1, excluding the possibility of Taq polymerase inhibition. Representative electrophoresis patterns obtained by the fluorescence-based TRAP analysis of two high-grade lymphomas with strong telomerase activities, one morphologically normal mucosa adjacent to the lymphoma with weak activity, and one low-grade MALT lymphoma without the activity are shown in Fig. 1. Quantitative values for the specimens evaluated in this study are summarized in Fig. 2, for a comparison between each of the disease entities.

Telomerase activity was clearly observed in all three specimens of gastric carcinoma (range, 16-129 TPG). No activity was observed in any of the adjacent normal mucosa. Strong activities (range, 43-332 TPG) comparable to those of carcinoma specimens, were identified in four of five surgically resected specimens (three with diffuse large-cell type and one with follicular lymphoma) and two of two biopsy specimens of highgrade lymphoma. No activity was observed from the resected specimen of the remaining one patient, who 
Table 1. Telomerase activity of MALT lymphoma and $H$. pylori-associated gastritis before and after eradication of $H$. pylori

\begin{tabular}{|c|c|c|c|c|c|c|}
\hline Case & $\begin{array}{l}\text { H. pylori prior } \\
\text { to eradication }\end{array}$ & $\begin{array}{l}\text { Grade prior to } \\
\text { eradication }\end{array}$ & $\begin{array}{l}\text { Grade after } \\
\text { eradication }\end{array}$ & $\begin{array}{l}\text { Telomerase activity } \\
\text { prior to eradication }\end{array}$ & $\begin{array}{l}\text { Telomerase activity } \\
\text { after eradication }\end{array}$ & $\begin{array}{c}\text { Telomerase activity } \\
\text { at surgery }\end{array}$ \\
\hline 1 & + & 5 & 4 & 0 & 0 & 0 \\
\hline 2 & + & 5 & 3 & 18 & 0 & Not performed \\
\hline 3 & + & 5 & 5 & 9 & 0 & 0 \\
\hline 4 & + & 5 & 2 & 39 & 0 & Not performed \\
\hline 5 & + & 5 & 3 & 0 & 0 & Not performed \\
\hline 6 & - & 5 & 5 & 0 & Not determined & Not performed \\
\hline 7 & + & 5 & 2 & 0 & Not determined & Not performed \\
\hline 8 & + & 3 & 2 & 0 & 0 & Not performed \\
\hline 9 & + & 3 & 3 & 0 & 0 & Not performed \\
\hline
\end{tabular}

MALT, mucosa-associated lymphoid tissue

H. pylori was successfully eradicated in all patients who were $H$. pylori-positive prior to antibiotic therapy

Histopathologic grades are determined as described by Wotherspoon et al. [7] in which Grades 4 and 5 are MALT lymphoma

Telomerase activity was quantitated with endoscopic biopsy specimens by fluorescence-based TRAP analysis

Only two patients (cases 1 and 3) underwent surgery; for these two, resected specimens were also evaluated with TRAP analysis

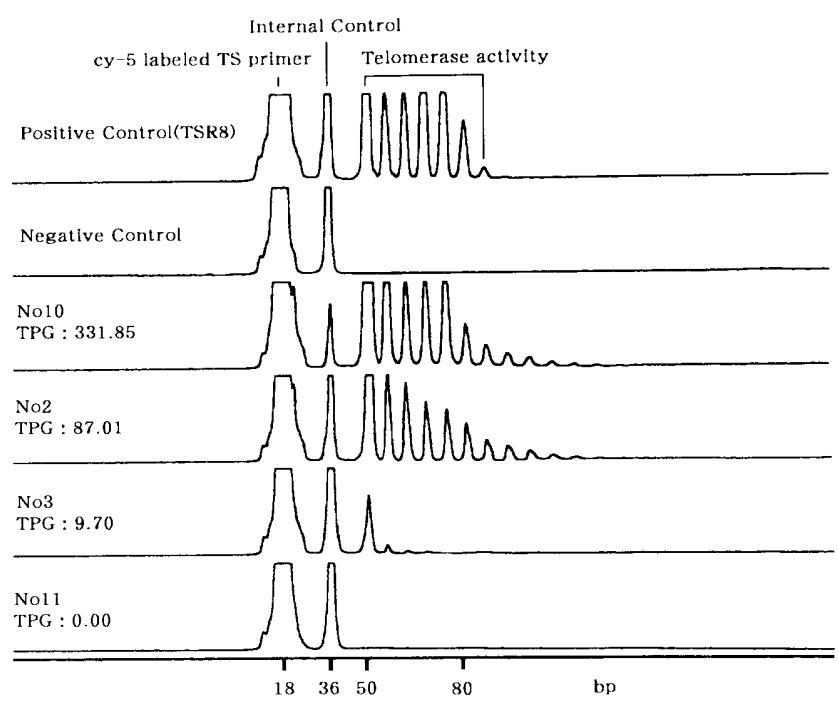

Fig. 1. Representative electrophoresis patterns of telomerase activity obtained by fluorescence-based telomeric amplitication assay protocol (TRAP) analysis. The first peak is attributed to $C y$-5-labeled telomerase substrate $(T S)$ primer. The second peak, at $36 \mathrm{bp}$, is the internal control, TSK1. Telomerase activity was characterized by a shark-tooth pattern starting from the third peak at $50 \mathrm{bp}$. From top to bottom, Positive control; negative control (3-[(3cholamidopropyl)-dimethylammonio]-1-propare-sulfonate; CHAPS lysis buffer); tumor tissue from case 1 with high-grade lymphoma (diffuse large-cell type); tumor tissue from case 2 with high-grade lymphoma (diffuse large-cell type); morphologically normal mucosa from case 2; tumor tissue from case 3 with low-grade mucosa-associated lymphoid tissue (MALT) lymphoma. TPG, Total product generated (units)

had high-grade lymphoma of the diffuse large-cell type. In two of the patients with telomerase-positive highgrade lymphoma, telomerase activities were also observed in the morphologically normal mucosa. These patients were found to have a positive surgical margin,

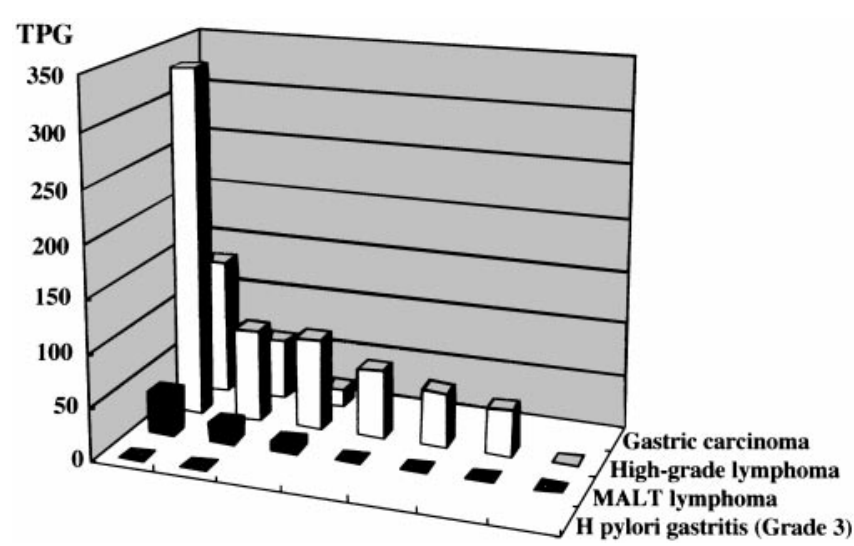

Fig. 2. Telomerase activity of surgically resected and biopsy specimens from primary gastric lymphoma and other gastric lesions. The enzymatic activity was quantified by fluorescencebased TRAP analysis. The biopsy samples of MALT lymphoma and Helicobacter pylori-related gastritis presented in this Fig. were taken endoscopically prior to the eradication of H. pylori

despite intraoperative macroscopic scrutiny of the mucosal surface, and the positive telomerase activities are probably attributable to microscopic involvement of the mucosa with neoplastic cells.

The telomerase activities in low-grade MALT lymphoma are summarized in Table 1 . Relatively weak telomerase activity (range, 9-39 TPG) was detected in three of the seven patients with low-grade MALT lymphoma prior to the eradication of H. pylori. No activity was observed in any of the adjacent mucosa from these seven patients. All seven patients were then given the antibiotics for the eradication of $H$. pylori, and telomerase activities were reevaluated in five patients. Telomerase activity was not detected in any of these patients following the eradication of $H$. pylori. Of these, 
two patients had no regression in terms of endoscopic and microscopic finding and underwent surgery. No telomerase activity had been observed in these patients from the biopsy specimens obtained after $H$. pylori eradication, and the activity remained undetectable in the surgically resected specimens, despite the histopathologic confirmation of MALT lymphoma. Telomerase activity was not observed in the biopsy specimens from the two patients with grade 3 gastritis.

\section{Discussion}

Telomerase activity has been useful for distinguishing between benign and malignant neoplasms of various origins [16-18]. In gastric neoplasms, however, precancerous lesions, including some gastric adenomas and intestinal metaplasias, reportedly express telomerase activity [22], in addition to carcinoma tissues, indicating that reactivation of telomerase may occur at an early stage of carcinogenesis. This finding prompted us to investigate whether telomerase activity becomes evident during the process of $H$. pylori infection, which causes gastritis and eventually leads to the evolution of low-grade MALT lymphoma of the stomach. However, given that telomerase activity is observed in benign lymphoid tissues [23,24], and even in normal B lymphocytes [25], whether telomerase activity can be used to differentiate between malignant lymphoid proliferations and reactive states was of considerable concern. On the other hand, high-grade lymphomas are reported to exhibit higher levels of telomerase than lowgrade ones in non-Hodgkin's lymphoma [23]. Further, telomerase activity in normal B-cells was found to be significantly weaker than that in neoplastic cells [25]. Telomerase activity was also shown to correlate with the prognosis of malignant lymphoma of the central nervous system [19]. These findings suggest the possibility that malignant lymphoproliferative lesions and reactive states may be distinguished by the quantitative evaluation of telomerase activity.

In the current study, telomerase activity was detected in six of seven specimens of high-grade lymphoma. Levels of the enzymatic activity in these six specimens were higher than or comparable to levels in three gastric carcinoma tissues which had been evaluated as referential controls in this study. All patients with highgrade lymphoma presented with endoscopically evident gastric tumor and had nodal and/or distant metastasis, including the patient with diffuse large-cell type lymphoma which somehow lacked telomerase activity. This lesion consisted of high-grade and low-grade components, as is sometimes the case with primary gastric lymphoma [9], and there is a remote possibility that the representative specimen was sampled from a portion predominantly infiltrated with the low-grade component which was deficient in telomerase activity.

Unlike other findings in precancerous lesions [22], the two patients in our study with pre-neoplastic grade 3 gastritis, with endoscopic findings compatible with MALT lymphoma and apparently associated with H. pylori infection, were found to be deficient in telomerase activity. Moreover, the activity was not observed in any of the mucosa adjacent to the seven lesions of low-grade MALT lymphoma, despite the possibility that these patients had various degrees of $H$. pylori-associated gastritis. Although telomerase activity was detectable in three of seven specimens of low-grade MALT lymphoma, the levels of telomerase activity in these specimens were generally weak compared with those in the high-grade lymphoma described above. Furthermore, the activity in these three patients with MALT lymphoma became undetectable shortly after the eradication of $H$. pylori, indicating that loss of telomerase activity occurs as an early event during the process of regression. These findings are in sharp contrast with findings of B-cell monoclonality, which was detected in $79 \%$ of patients with chronic gastritis who eventually developed MALT lymphoma [26], while B-cell monoclonality remained detectable in some lesions after the histopathologic regression of MALT lymphoma following $H$. pylori eradication therapy [10].

Of various telomerase-related components reported to date, only telomerase enzymatic activity was evaluated in this study, because: (1) the mumber of endoscopic biopsy specimens available was limited, (2) telomerase activity was shown to correlate with the expression of the key-component h-TERT [27], and (3) this study was intended, in part, to evaluate the clinical benefit of measurement using the fluorescence-based TRAP described above. The method used in this study is already accessible to endoscopists and pathologists throughout Japan, and can be swiftly introduced to daily practice once the clinical relevance of the assay is recognized. One major weakness of this method, though, is that the samples will have to be snap-frozen to be evaluated quantitatively, meaning that the evaluation cannot be performed for archival paraffinembedded specimens.

In summary, telomerase activity was detected more frequently and at higher levels in high-grade lymphomas compared with low-grade ones, as has been reported in nodal non-Hodgkin's lymphoma. This activity was detected in some low-grade MALT lymphomas of the stomach, but was undetectable before the establishment of the neoplasm and after $H$. pylori eradication therapy. The PCR detection of B-cell monoclonality reportedly lacks specificity, while being a sensitive indicator of gastritis with a high risk of 
developing into MALT lymphoma. In contrast, telomerase activity evaluated with the fluorescencebased TRAP analysis may have a role in confirming the histologic diagnosis of primary gastric lymphoma. A larger scale prospective study is warranted to further assess the clinical relevance of this enzymatic assay in the diagnosis and follow-up of low-grade MALT lymphoma and $H$. pylori-associated gastritis.

\section{References}

1. Kodera Y, Yamamura Y, Nakamura S, Shimizu Y, Torii A, Hirai T, et al. The role of radical gastrectomy with systematic lymphadenectomy for the diagnosis and treatment of primary gastric lymphoma. Ann Surg 1998;227:45-50.

2. Sano T, Sasako M, Kinoshita T, Katai H, Maruyama K, Takenaka $\mathrm{T}$, et al. Total gastrectomy for primary gastric lymphoma at stages IE and IIE: a prospective study of 50 cases. Surgery 1997;121:5015 .

3. Maor MH, Velasquez WS, Fuller LM, Silvermintz KB. Stomach conservation in stages IE and IIE gastric non-Hodgkin's lymphoma. J Clin Oncol 1990;8:266-7.

4. Koch P, Grothaus-Pinke B, Hiddemann W, Willich N, Reers B, del Valle F. Primary lymphoma of the stomach: three-year results of a prospective multicenter study. Ann Oncol 1997;8:S85-8.

5. Isaacson PG, Spencer J, Finn T. Primary B-cell gastric lymphoma. Hum Pathol 1986;17:72-82.

6. Isaacson PG, Wright DH. Extranodal malignant lymphoma arising from mucosa-associated lymphoid tissue. Cancer 1984;53: 2515-24.

7. Wotherspoon AC, Doglioni C, Diss TC, Pan L, Moschini A, de Boni M, et al. Regression of primary low-grade B-cell gastric lymphoma of mucosa-associated lymphoid type after eradication of Helicobacter pylori. Lancet 1993;342:575-7.

8. Bayerdorffer E, Neubauer A, Rudolph B, Thiede C, Lehn N, Eidt $\mathrm{S}$, et al. Regression of primary gastric lymphoma of mucosa associated lymphoid tissue type after cure of Helicobacter pylori infection. Lancet 1995;345:1591-4.

9. Cogliatti SB, Schmid U, Schumacher U, Eckert F, Hansmann M-L, Hedderich J, et al. Primary B-cell gastric lymphoma: a clinicopathologicalogy study of 145 patients. Gastroenterology 1991;101:1159-70.

10. Savio A, Franzin G, Wotherspoon AC, Zambosi G, Negrini R, Buffoli F, et al. Diagnosis and posttreatment follow-up of Helicobacter pylori-positive gastric lymphoma of mucosaassociated lymphoid tissue: histology, polymerase chain reaction, or both? Blood 1996;87:1255-60.

11. Blackburn EH. Structure and function of telomeres. Nature 1991;350:569-73.
12. Kim NW, Piatyzek MA, Prowse KR, Harley CB, West MD, Ho PLC, et al. Specific association of human telomerase activity with immortal cells and cancer. Science 1994;266:2011-5.

13. Hiyama E, Yokoyama T, Tatsumoto N, Hiyama K, Imamura Y, Murakami Y, et al. Telomerase activity in gastric cancer. Cancer Res 1995;55:3258-62.

14. Taga S, Osaki T, Ohgami A, Imoto H, Yasumoto K. Prognostic impact of telomerase activity in non-small cell lung cancers. Ann Surg 1999;230:715-20.

15. Chadeneau C, Hay K, Hirte HW, Gallinger SS, Bacchetti S. Telomerase activity associated with acquisition of malignancy in human colorectal cancer. Cancer Res 1995;55:2533-6.

16. Teng L, Tucker O, Malchoff C, Vaughan ED, Jacobson J, Fahey III TJ, et al. Telomerase activity in the differentiation of benign and malignant adrenal tumors. Surgery 1998;124:1123-7.

17. Sakurai S, Fukayama M, Kaizaki Y, Saito K, Kanazawa K, Kitamura M, et al. Telomerase activity in gastrointestinal stromal tumors. Cancer 1998;83:2060-6.

18. Umbricht CB, Saji M, Westra WH, Udelsman R, Zeiger MA, Sukumar S. Telomerase activity: a marker to distinguish follicular thyroid adenoma from carcinoma. Cancer Res 1997;57:21447.

19. Harada K, Kurisu K, Arita K, Sadamoto T, Tahara H, Tahara E, et al. Telomerase activity in central nervous system malignant lymphoma. Cancer 1999;86:1050-5.

20. Hisatomi H, Nagao K, Komatsu H. Quantification of telomerase activity in human liver tissues by fluorescence-based TRAP analysis. Hepatol Res 1997;7:35-42.

21. Aden DP, Fogel A, Plotkin S, Damajnov I, Knowles BB. Controlled synthesis of HbsAg in a differentiated human liver carcinoma derived cell line. Nature 1979;282:615-6.

22. Tahara H, Kuniyasu H, Yokozaki H, Yasui W, Shay JW, Ide T, et al. Telomerase activity in preneoplastic and neoplastic gastric and colorectal lesion. Clin Cancer Res 1995;1:1245-51.

23. Norrback K-F, Dahlenborg K, Carlsson R, Roos G. Telomerase activation in normal B lymphocytes and non-Hodgkin's lymphomas. Blood 1996;88:222-9.

24. Brousset P, Saati TA, Chaouche N, Zenou R-C, Schlaifer D, Chittal S, et al. Telomerase activity in reactive and neoplastic lymphoid tissues: infrequent detection of activity in Hodgkin's disease. Blood 1997;89:26-31.

25. Trentin L, Ballon G, Ometto L, Perin A, Basso U, Chieco-Bianchi $\mathrm{L}$, et al. Telomerase activity in chronic lymphoproliferative disorders of B-cell lineage. Br J Haematol 1999;106:662-8.

26. Nakamura S, Aoyagi K, Furuse M, Suekane H, Matsumoto T, Yao $\mathrm{T}$, et al. B-cell monoclonality precedes the development of gastric MALT lymphoma in Helicobacter pylori-associated chronic gastritis. Am J Pathol 1998;152:1271-9.

27. Gonzalez-Quevedo R, De Juan C, Massa MJ, Sanchez-Pernaute A, Torres A, Balibrea JL, et al. Detection of telomerase activity in human carcinomas using a TRAP-ELISA method: correlation with hTR and hTERT expression. Int J Oncol 2000;16:6238. 